\title{
Collective and fractal properties of pion jets in the four-velocity space at intermediate energies
}

\author{
V.A. Okorokov * A.K. Ponosov, and F.M. Sergeev \\ National Research Nuclear University "MEPhI", Kashirskoe Shosse 31, 115409 Moscow, Russia
}

(Dated: April 30, 2022)

\begin{abstract}
Experimental results are presented for study of collective and fractal properties of soft pion jets in the space of relative four-dimensional velocities. Significant decreasing is obtained for mean square of second particle distances from jet axis for pion-proton interactions at initial energies $\sim 3 \mathrm{GeV}$ in comparison with hadron-nuclear collisions at close energies. The decreasing results in power dependence of distance variable on collision energy for range $\sim 2-4 \mathrm{GeV}$. The observation allows us to estimate the low boundary of manifestation of color degree of freedom in pion jet production. Cluster dimension values were deduced for pion jets in various reactions. Fractional values of this dimension indicate on the manifestation of fractal-like properties by pion jets. Changing of mean kinetic energy of jet particles and fractal dimension with initial energy increasing is consistent with suggestion for presence of color degrees of freedom in pion jet production at intermediate energies.

PACS 13.75.-n
\end{abstract}

\section{INTRODUCTION}

Investigation of the mixed phase, transition from meson-nucleon to quark-gluon degrees of freedom is now one of the most actual and important tasks of the world program of researches in the field of strong interactions. Experimental study of hadron-hadron and hadron-nuclear interactions can give the important information concerning manifestation of new (color) degrees of freedom. In various fields of physics the beginning of manifestation of new degrees of freedom and transition processes are accompanied by presence of self-affine, fractal features for collective effects. Thus, study of collective and geometrical (fractal) properties, in particular, soft hadron jets at intermediate energies can give the new important information concerning hadronization mechanisms, non-perturbative physics and transition to manifestation of color degrees of freedom in collective phenomena.

A relativistic invariant method for investigation of collective effects in multiparticle production processes of the type I + II $\rightarrow 1+2+\ldots$ was proposed in [1]. Features of this method for events with two jets were in detail considered in [2, 3]. In this case the secondary particles emitted in the regions of target and beam fragmentation can be separated by means of a relativistic invariant variable $X_{1}=0.1-0.2$. One of the main variables of the method is $b_{k}=-\left(V-U_{k}\right)^{2}-$ a square of distance $k$-th particle from an axis of jet $V$ in the space of four-dimensional velocities $U_{i}=P_{i} / m_{i}, i=\mathrm{I}, \mathrm{II}, 1,2 \ldots$ [1]. It should be stressed that in the framework of this approach the range of values $10^{-2} \leq b_{k} \sim 1$ corresponds to transition from domination of meson-nucleon degrees of freedom to manifestation of internal structure of initial particles and, thus, to quark-gluon degrees of freedom in processes of production of secondary particles.

In [4] it was suggested to study the geometrical properties of pion jets in four-velocity space by means of cluster dimension, $D$, defined by a relation between number of particles in jet, $N\left(b_{k}\right)$, and its radius $N\left(b_{k}\right) \propto b_{k}^{D / 2}$.

In this paper, collective and geometrical properties of soft pion jets have been studied using the space of relative four-dimensional velocities for following reactions:

$$
\begin{gathered}
\pi^{-}+\mathrm{p} \rightarrow \mathrm{N}+k \pi^{-}+l \pi^{+}+j \pi^{0}, \quad k=2, l=1,2, j \geq 0, \quad P_{0}=(3.93 \pm 0.01) \mathrm{GeV} / \mathrm{c} ; \\
\pi^{+}+\mathrm{p} \rightarrow \begin{cases}\mathrm{N}+K^{+}\left(K^{0}\right)+\bar{K}^{0}\left(K^{-}\right)+k \pi^{-}+l \pi^{+}+j \pi^{0}, & P_{0}=(3.90 \pm 0.30) \mathrm{GeV} / \mathrm{c} \\
\mathrm{B}_{s}+K^{0,+}+k \pi^{-}+l \pi^{+}+j \pi^{0}, & k, j=0,1, l \geq 0 ;\end{cases} \\
\pi^{+}+\mathrm{p} \rightarrow \mathrm{N}+k \pi^{-}+l \pi^{+}+j \pi^{0}, \quad k=1,2, l=2-4, j=0,1, \quad P_{0}=(4.23 \pm 0.08) \mathrm{GeV} / \mathrm{c} ; \\
\pi^{-}+\left(\mathrm{C}_{2} \mathrm{~F}_{5} \mathrm{Cl}_{3}\right) \rightarrow \Lambda^{0}+K^{0}+k \pi^{-}+l \pi^{+}+m \mathrm{p}+X, \quad k, l, m>0, \quad P_{0}=(3.86 \pm 0.04) \mathrm{GeV} / \mathrm{c} \\
\pi^{-}+\mathrm{Ne} \rightarrow k \pi^{-}+l \pi^{+}+m \mathrm{p}+X, \quad k, l, m>0, \quad P_{0}=(6.20 \pm 0.10) \mathrm{GeV} / \mathrm{c} ;
\end{gathered}
$$

*Electronic address: VAOkorokov@mephi.ru; okorokov@bnl.gov 
Here $P_{0}$ - beam momentum, $\mathrm{N}=\mathrm{p}, \mathrm{n}, \mathrm{B}_{s}=\Lambda^{0}, \Sigma^{0, \pm}$ are final state strange baryon, moreover $l=1,2$ at $\mathrm{B}_{s}=\Lambda^{0}$ and $l=0-3$ at $\mathrm{B}_{s}=\Sigma^{0, \pm}$ in the reaction (2). The technique of experiments and a selection criteria are described in detail in [3, 5]. The general statistics for reactions (11) - (5) is more than $1.9 \cdot 10^{5}$ events [6].

\section{COLLECTIVE PROPERTIES OF PION JETS}

Fig 1 shows dependencies of mean square of distance from axis of pion jet in the four-velocity space $-\left\langle b_{k}\right\rangle-$ on initial energy $\sqrt{s}$ (mean total energy of final hadron state in the c.m. frame, $W$, for $\bar{\nu} \mathrm{N}$ ) for secondary $\pi^{-}$mesons at cutoff $X_{1}=0.1(\mathrm{a}, \mathrm{b})$ and $0.2(\mathrm{c}, \mathrm{d})$ for various interactions.

At initial energies $\sqrt{s}>8$ the dependence $\left\langle b_{k}\right\rangle(\sqrt{s})$ has been fitted by logarithmic function

$$
\left\langle b_{k}\right\rangle=a_{1}+a_{2} \ln \left(s / s_{0}\right),
$$

where $s_{0}=1 \mathrm{GeV}^{2}$. Results of above fit are presented on Fig $1 \mathrm{~b}$,d (dashed line) and in Table I. Only experimental data are fitted at $X_{1}=0.1$ for energy range under considered. As seen the Lund model calculations for $\pi^{-} \mathrm{p}$ interactions at $40 \mathrm{GeV} / \mathrm{c}$ are in good agreement with experimental data, unlike results obtained by the model of homogeneously filled phase volume (Fig,1 b). The fit with taking into account results of Lund model calculations for $\pi^{-} \mathrm{p}$ at 40 and $360 \mathrm{GeV} / \mathrm{c}$ gives parameter values which coincide within errors with the values indicated in Table I at some improvement of fit quality $\left(\chi^{2} /\right.$ n.d.f. $\left.=4.53\right)$. The value of $a_{2}$ parameter in (6) coincides with zero within errors at $X_{1}=0.2$ in the energy domain $\sqrt{s}>8 \mathrm{GeV}$. Therefore data were fitted at the fixed value $a_{2}=0$, that allows to obtain better quality of approximation. However, it is impossible to exclude unambiguously the weak logarithmic growth for $\left\langle b_{k}\right\rangle$ in accordance with (6) taking into account insignificant ensemble of accessible experimental data. Earlier in [3] statistically reasonable description by function (6) had been obtained for experimental data for hadron-hadron and $\bar{\nu} \mathrm{N}$ reactions, and also for hadron-nuclear interactions, at $3.5<\sqrt{s}<9 \mathrm{GeV}$ (solid lines on Fig $1 \mathrm{~b}, \mathrm{~d}$ ). Thus, dependence $\left\langle b_{k}\right\rangle(\sqrt{s})$ supposes universal approximation (6) at $\sqrt{s}>3.5 \mathrm{GeV}$ for a wide class of interactions at any values $X_{1}$ under study.

The hypothesis has been suggested in [3] about change of dynamic regime at $\sqrt{s}<3-4 \mathrm{GeV}$, reflected in the behaviour of underlying dependence. This energy domain has been investigated in more details in the present paper. Dependencies $\left\langle b_{k}\right\rangle(\sqrt{s})$ are shown on Fig $1 \mathrm{a}, \mathrm{b}$ in collision energy range $\sqrt{s}<4 \mathrm{GeV}$ with taking into account of uncertainties of initial momenta for various types of interactions with exception of hadron-nuclear ones at $X_{1}=0.1$ and 0.2 , respectively. The results obtained here for a pion-proton reactions (11) and (2) indicate clearly on the change of behaviour of dependence $\left\langle b_{k}\right\rangle$ at $\sqrt{s} \sim 3 \mathrm{GeV}$ (Fig,1), confirming the our earlier suggestion [3]. Results for symmetric nuclear collisions agree well with the general tendency (Fig 1 $\mathrm{h}, \mathrm{b})$ at $X_{1}=0.1$. Dependence of $\left\langle b_{k}\right\rangle$ values on $\sqrt{s}(W)$ has been fitted by following power function at $\sqrt{s}<4 \mathrm{GeV}$ :

$$
\left\langle b_{k}\right\rangle=a_{1}\left(\sqrt{s / s_{0}}-a_{2}\right)^{a_{3}}, \quad \sqrt{s / s_{0}} \geq a_{2} .
$$

The results of the above fit are shown on Fig! (dotted lines) and in Table I. In [3, 6] it has been shown, that properties of soft pion jets depend on region of fragmentation in the energy domain under study. This effect leads to significant distinction of corresponding $\left\langle b_{k}\right\rangle$ values. Therefore sharp behaviour of dependence $\left\langle b_{k}\right\rangle(\sqrt{s})$ and disorder of experimental points result in statistically unacceptable fit quality. However, as seen from Fig 1 power function agrees well enough with experimental data at all values of $X_{1}$ at qualitative level.

Experimental data samples were fitted for target fragmentation region (curves 1 on Fig [1,, $\mathrm{c}$ ) and for beam fragmentation one (curves 2 on Fig[1a,c) separately. The values of fit parameters are listed in Table [I] Substantial improvement of fit quality is observed for any fragmentation region and values of $X_{1}$. The data samples at $X_{1}=0.2$ were fitted at the fixed value of parameter $a_{3}=0.5$ taking into account the results obtained at soft cutoff and volumes of accessible data samples at $X_{1}=0.2$. Thus, dependence $\left\langle b_{k}\right\rangle(\sqrt{s})$ is described by power function (17) at $\sqrt{s}<4 \mathrm{GeV}$ for studied types of interactions $(\mathrm{hh}, \bar{\nu} \mathrm{N}, \mathrm{AA})$ both for separate samples for various fragmentation regions and for total data ensemble. It is important to note, that in general the power behaviour is one of the characteristic features of transition domain in which new degrees of freedom of investigated system begin to be manifest. Value of parameter $a_{2}$ can be put in correspondence with the energy (in $\mathrm{GeV}), \sqrt{s_{c}}$, at which the internal structure of interacting particles, i.e. new degrees of freedom, begins to manifest in the pion jet production. Thus, the estimation is obtained, that new (color) degrees of freedom begin to be manifest experimentally at $\sqrt{s_{c}} \simeq 2.5-2.8 \mathrm{GeV}$ in jet production of pions.

Dependencies $\left\langle b_{k}\right\rangle(\sqrt{s})$ are presented at Fig 2 for secondary neutral strange particles, $K^{0}$ mesons $(\mathrm{a}, \mathrm{c})$ and $\Lambda^{0}$ hyperons (b,d), at various values of $X_{1}$. As seen, usually, $\left\langle b_{k}\right\rangle$ value is smaller for target fragmentation region, than corresponding $\left\langle b_{k}\right\rangle$ for beam fragmentation one. The reactions (22) and (4) investigated here with absence of strange particles in an initial state and with its production in a final state can be considered as additional argument in favour 
TABLE I: Fit parameters for dependence $\left\langle b_{k}\right\rangle(\sqrt{s})$.

\begin{tabular}{|l|c|c|}
\hline Fit parameter & $X_{1}=0.1$ & $X_{1}=0.2$ \\
\hline hh-, hA-interactions $(\sqrt{s}>8 \mathrm{GeV})$ \\
\hline$a_{1}$ & $3.7 \pm 0.2$ & $4.88 \pm 0.05$ \\
$a_{2}$ & $0.12 \pm 0.05$ & 0.0 (fixed) \\
$\chi^{2} /$ n.d.f. & 7.20 & 1.71 \\
\hline hh-, AA-interactions $(\sqrt{s}<4 \mathrm{GeV})$ \\
\hline$a_{1}$ & $3.69 \pm 0.02$ & $3.63 \pm 0.05$ \\
$a_{2}$ & $2.76 \pm 0.01$ & $2.51 \pm 0.03$ \\
$a_{3}$ & $0.49 \pm 0.01$ & $0.40 \pm 0.01$ \\
$\chi^{2} /$ n.d.f. & 133 & 9.25 \\
\hline
\end{tabular}

TABLE II: Parameter values for $\left\langle b_{k}\right\rangle(\sqrt{s})$ at $\sqrt{s}<4 \mathrm{GeV}$. Separated fitting for target and beam fragmentation regions.

\begin{tabular}{|c|c|c|c|c|}
\hline \multirow{2}{*}{$X_{1}$} & \multicolumn{5}{|c|}{ Fit parameters } \\
\cline { 2 - 5 } & $a_{1}$ & $a_{2}$ & $a_{3}$ & $\chi^{2} /$ n.d.f. \\
\hline \multicolumn{5}{|c|}{ target fragmentation } \\
\hline 0.1 & $3.79 \pm 0.03$ & $2.82 \pm 0.02$ & $0.52 \pm 0.05$ & 5.70 \\
0.2 & $3.52 \pm 0.07$ & $2.46 \pm 0.04$ & 0.5 (fixed) & 0.09 \\
\hline \multicolumn{5}{|c|}{ beam fragmentation } \\
\hline 0.1 & $2.65 \pm 0.13$ & $2.43 \pm 0.04$ & $0.50 \pm 0.35$ & 5.19 \\
0.2 & $4.6 \pm 1.9$ & $2.5 \pm 0.3$ & 0.5 (fixed) & 2.29 \\
\hline
\end{tabular}

of manifestation of quark degrees of freedom at $\left\langle b_{k}\right\rangle \sim 1$ at intermediate energies. Accessible samples of results is much less in case of neutral strange particles, than for $\pi^{-}$mesons. Therefore it is possible to study qualitative behaviour of $\left\langle b_{k}\right\rangle(\sqrt{s})$ at Fig, 2 only. For $K^{0}$ mesons as well as for secondary pions, the increasing of $\left\langle b_{k}\right\rangle$ at growth of initial energy is observed at any $X_{1}$ under considered (Fig, 2 a,c). One can see that behaviour of $\left\langle b_{k}\right\rangle(\sqrt{s})$ for $\Lambda^{0}$ hyperons at $X_{1}=0.1$ (Fig 2 b) is similar to corresponding dependence for $K^{0}$ mesons (Fig 2 a), however $\left\langle b_{k}\right\rangle$ values for $\Lambda^{0}$ do not depend on collision energy practically at more hard cutoff $X_{1}=0.2$ (Fig $2 \mathrm{~d}$ ).

The dependence of $\left\langle b_{k}\right\rangle$ value on particle mass has been investigated for secondary $\pi^{0, \pm}, K^{0, \pm}, \Lambda^{0}$ and $\Sigma^{0, \pm}$ particles from reaction (2). The relativistic invariant variable $X_{1}$ was used for separation of various fragmentation region for all particle types with exception of $\Sigma$ hyperons. The Feynman variable $x_{\mathrm{F}}$ was used for separation of more heavy $\Sigma$ particles on fragmentation region. Significant decreasing of mean square of distance from jet axis is observed for kaons in comparison with pions with the weaker decreasing at further increasing of particle mass that agrees with the behaviour of similar dependence at higher energies $\sqrt{s} \simeq 8.7 \mathrm{GeV}$ qualitatively [6].

The important characteristic of applied approach is the mean kinetic energy of particles in jet in the its rest frame (this parameter is called "temperature" often), $\left\langle T_{k}\right\rangle$, which is calculated on the basis of fitting of invariant $F\left(b_{k}\right)$ distributions [7]. Fig 3 shows dependencies $\left\langle T_{k}\right\rangle(\sqrt{s})$ for pion jets in the target fragmentation region (a,c) and for beam fragmentation one $(\mathrm{b}, \mathrm{d})$. The published distributions $1 / N d N / d b_{k}[10]$ have been used for an estimation of $\left\langle T_{k}\right\rangle$ in case of $\bar{p}$, pp, $\pi^{-} \mathrm{C}$ interactions, results for $\pi^{-} \mathrm{p}$ reaction at $40 \mathrm{GeV} / \mathrm{c}$ are taken from [11]. Accessible experimental data allow to study behaviour of $\left\langle T_{k}\right\rangle(\sqrt{s})$ at a qualitative level. The increasing of $\left\langle T_{k}\right\rangle$ is observed with growth of initial energy both for hadron-hadron and for hadron-nuclear collisions. It is important to note significant increasing of $\left\langle T_{k}\right\rangle$ in a narrow range of $\sqrt{s}$ for reactions (1), (3), and much smoother growth at the further increasing of initial energy. Additional experimental investigations are necessary for interactions of various types at $\sqrt{s} \sim 3-20 \mathrm{GeV}$, however, the obtained results do not contradict the hypothesis above concerning manifestation of new degrees of freedom in processes of soft pion jet production at $\sqrt{s} \sim 3 \mathrm{GeV}$.

The results obtained for $\pi^{-}$mesons from jets (clusters) of secondary particles of various types in proton-nuclear and in nuclear-nuclear interactions with using of normalized relative four-velocity space for central region and for beam fragmentation [9] are agreed with behaviour of $\left\langle T_{k}\right\rangle(\sqrt{s})$ at $X_{1}=0.1$ for beam fragmentation (Fig[3b) qualitatively.

\section{CLUSTER DIMENSIONS FOR JETS}

Fig 4 shows cluster dimension $D$, calculated for secondary $\pi^{-}$-mesons, versus collision energy $\sqrt{s}$ for hadron-hadron and hadron-nuclear interactions at $X_{1}=0.1(\mathrm{a}, \mathrm{b})$ and at $X_{1}=0.2(\mathrm{c}, \mathrm{d})$ for region of target fragmentation (a,c) and for beam fragmentation $(\mathrm{b}, \mathrm{d})$. Experimental data used for calculation of cluster dimension are taken from [10] for $\bar{p} p$ and pp interactions, from [10,11] - for $\pi^{-}$p and $\pi^{-} \mathrm{C}$ at $40 \mathrm{GeV} / \mathrm{c}$. Cluster dimension shows fractional value for all reactions under study with exception of hadron-nuclear interactions (4) and (5), that allows to suggest the presence of indication on manifestation of fractal-like properties of pion jets in collision energy range under considered. The parameter $D$ have values close to the integer ones within error bars for reactions (4) and (5), excepting for $\pi^{-}$Ne reaction for beam fragmentation at more hard cutoff $X_{1}=0.2$. Accessible experimental data allow to study the behaviour of $D(\sqrt{s})$ and some features of the dependence only at a qualitative level. At any used $X_{1}$ cluster dimension for reactions (4) and (5) is close to the integer value within larger errors as indicated above, results obtained for hadron-nuclear reactions show increasing of $D$ with growth of initial energy. The hadron-hadron interactions show a different behaviour of $D(\sqrt{s})$ in various range of initial energies for region of target fragmentation. Significant growth of cluster dimension 
is observed at small increasing of collision energy for reactions (11) and (3), the further increasing of $\sqrt{s}$ leads to much weaker growth of $D$ with the subsequent reaching of a constant at $X_{1}=0.1$ (Fig, $4 \mathrm{a}$ ) or to absence of changes at all for $X_{1}=0.2$ (Fig,4 $)$. The dependencies $D(\sqrt{s})$ for beam fragmentation region (Fig, 40,d) do not contradict with behaviour of corresponding dependencies described above for a target fragmentation. The observed behaviour of cluster dimension versus collision energy and sharp amplification of manifestation of fractal-like properties, which is one of the important and characteristic features for occurrence of new degrees of freedom, does not contradict a hypothesis about presence of transition energy range to experimentally noticeable manifestation of quark degrees of freedom in hadron jet production at $\sqrt{s} \sim 3 \mathrm{GeV}$.

There is an intensive production of meson resonances in the energy range under study. Influence of resonances on cluster dimension of pion jets has been investigated for following exclusive channels $\pi^{-}+\mathrm{p} \rightarrow \mathrm{p}+\pi^{+}+2 \pi^{-}, \pi^{+}+\mathrm{p} \rightarrow$ $\mathrm{p}+2 \pi^{+}+\pi^{-}$of reactions (11) and (3), respectively. For jets (clusters) of particles with identical masses there is the unambiguous relation between $\left\langle b_{k}\right\rangle$ and jet effective mass [6, 12]. In consequence of this relation meson resonances, in particular, clear identified $\rho(700)$ and $a_{1}(1320)$, give rise to the normalized dependence of number of particles in jet on its radius, $N\left(b_{k}\right) / N_{\text {tot }}$, in value range $3 \leq b_{k}<4$ for beam fragmentation region. The influence of the specified resonances on cluster dimension is negligible for $\pi^{-} \mathrm{p}$ exclusive channel under study at soft condition of selection $X_{1}=0.1$ and significantly larger at hard cutoff. But the influence of these resonances is observable for corresponding $\pi^{+} \mathrm{p}$ channel at any $X_{1}$. The contribution from decay of meson resonances leads to significant growth of fractal cluster dimension of pion jets. Influence of resonances has been investigated in details for exclusive channels with one $\pi^{0}$ in reactions (11) and (3) also. However because of narrowness of $\eta$ and $\omega$ mesons it is possible to speak only about presence of indication on the influence of these resonances on cluster dimension.

\section{SUMMARY}

In conclusion, we summarize the main results of this study.

Values of $\left\langle b_{k}\right\rangle$ are significantly smaller for reactions (11) - (3) at $\sqrt{s} \sim 3 \mathrm{GeV}$ than those in other interactions at slightly higher energies, that leads to changing of dependence $\left\langle b_{k}\right\rangle(\sqrt{s})$ from logarithmic behaviour to power one. It is possible to assume, that this effect is caused by occurrence of experimentally noticeable manifestation of quark degrees of freedom in soft pion jet production and corresponding transition from the description of the such process at language of nucleon-meson degrees of freedom to using of color (quark-gluon) degrees of freedom. The behaviour of dependence $\left\langle T_{k}\right\rangle(\sqrt{s})$ for hadron-hadron interactions qualitatively confirms this hypothesis.

The cluster dimension of soft pion jets has been obtained for various reactions at $\sqrt{s} \sim 3-20 \mathrm{GeV}$ for the first time. The dimension shows fractional value for main part of reactions under considered that allows to suggest the presence of fractal-like properties for pion jets. Features of behaviour of dependence $D(\sqrt{s})$ obtained for hadronhadron reactions do not contradict the above suggestion of manifestation of new degrees of freedom at $\sqrt{s} \sim 3 \mathrm{GeV}$. Influence of meson resonances is observed on fractal dimensions of pion jets in beam fragmentation region, leading to significant increasing of $D$.

[1] A. M. Baldin, Doklady Akad. Nauk SSSR 222, 1064 (1975); 279, 1352 (1984).

[2] I. L. Kiselevich et al., Yad.Fiz. 57, 2225 (1994) [Phys. At. Nucl. (Engl. Transl.) 57, 2140].

[3] V. I. Mikhailichenko et al., Yad.Fiz. 62, 1787 (1999) [Phys. At. Nucl. (Engl. Transl.) 62, 1665].

[4] V. A. Okorokov et al., in Proceedings of the Scientific session MEPhI-2000. MEPhI, V.7, 2000, p. $218 ; 220$.

[5] V. Chaloupka et al., Phys. Lett. 44B, 211 (1973); M. G. Gornov et al., Yad.Fiz. 25, 606 (1977); 27, 1578 (1978); I. L. Kiselevich, PhD thesis(ITEP, Moscow, 1995).

[6] V.A. Okorokov in Proceedings of the XVIII International Baldin seminar on high energy physics problems. Dubna, JINR, V.I, 2008, p. 154.

[7] A. M. Baldin et al., JINR Rapid commun. N16, 24 (1986); A. M. Baldin, L. A. Didenko, Fortschr. Phys. 38, 261 (1990); B. A. Kulakov, Yu. Karachuk, JINR Rapid commun. N4, 45 (1997).

[8] A. M. Baldin et al., Preprint JINR, 1-85-820, Dubna (1985).

[9] N. Angelov et al., JINR Rapid commun. N2, 4 (1990).

[10] A. M. Baldin et al., Preprint JINR, E1-87-142, Dubna (1987); A. I. Malakhov et al., ECHAYA 38, 780 (2007).

[11] A. A. Baldin, A. M. Baldin, ECHAYA 29, 577 (1998).

[12] V. G. Grishin Kvarki $i$ adrony vo vzaimodeistviyakh chastits visokikh energii (Quarks and Hadrons in Interactions of High-Energy Particles). Moscow: Energoatomizdat, 1988. 


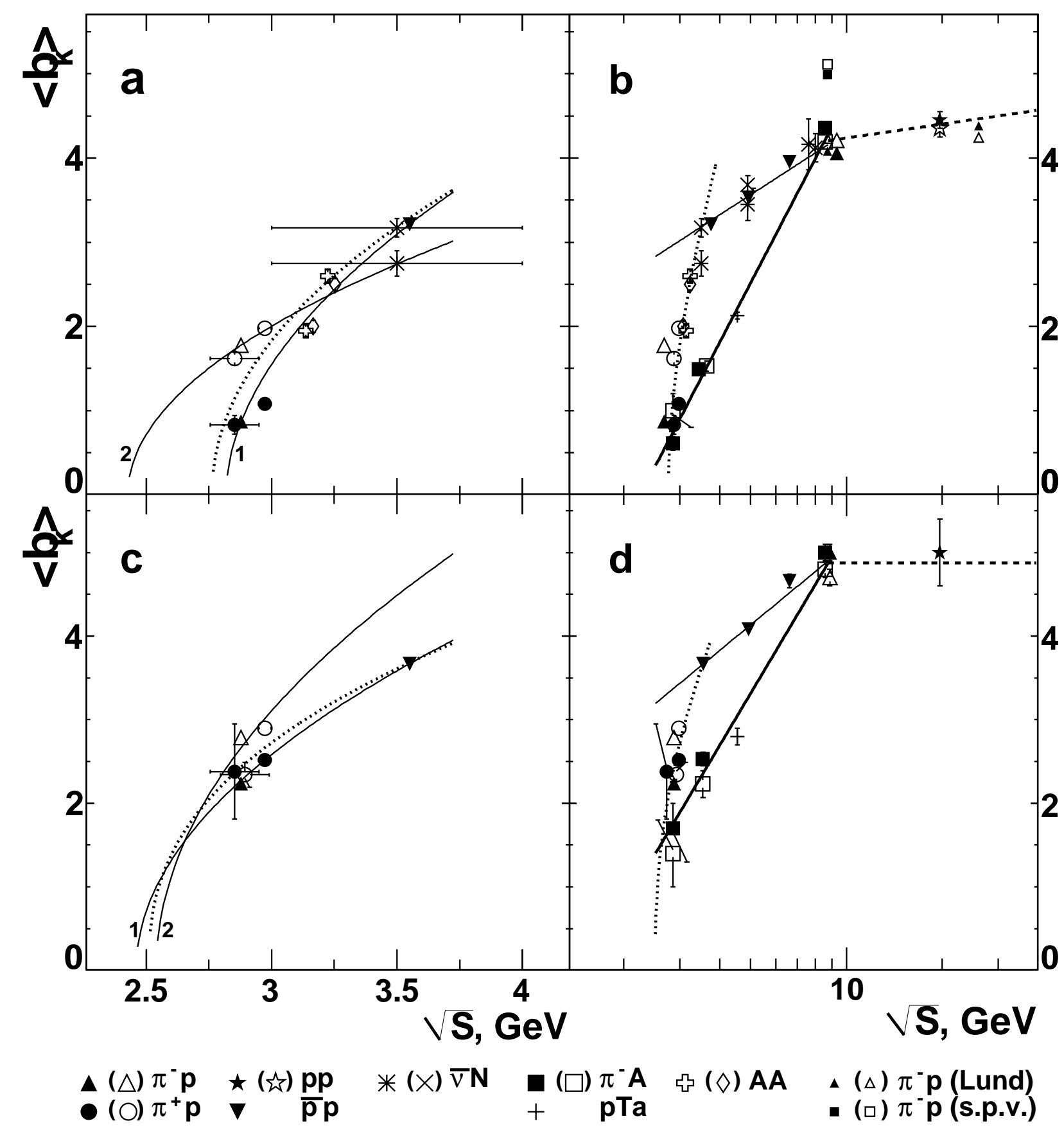

FIG. 1: Dependence of $\left\langle b_{k}^{\pi}\right\rangle$ on $\sqrt{s}(W)$ at $X_{1}=0.1(\mathrm{a}, \mathrm{b})$ and $X_{1}=0.2(\mathrm{c}, \mathrm{d})$. Experimental data for reactions (3) - (5) are from [2, [3, 6], for $\overline{\mathrm{p} p}$ at 5.7, 12 and $22.4 \mathrm{GeV} / \mathrm{c}, \bar{\nu} \mathrm{N}$ at $\mathrm{W}=3-4,4-6$ and $\geq 6 \mathrm{GeV}$, pp at $205 \mathrm{GeV} / \mathrm{c}, \pi^{-} \mathrm{p}$ at $40 \mathrm{GeV} / \mathrm{c}, \mathrm{pTa}+\mathrm{p}\left(\mathrm{C}_{3} \mathrm{H}_{8}\right)$ at $10 \mathrm{GeV} / \mathrm{c}, \pi^{-} \mathrm{C}$ at $40 \mathrm{GeV} / \mathrm{c}, \mathrm{CC}$ at $4.2 \mathrm{GeV} / \mathrm{c} / \mathrm{A}, \mathrm{MgMg}$ at $4.5 \mathrm{GeV} / \mathrm{c} / \mathrm{A}$, model calculations - from 7]. The curves are described in the main body of the text. 


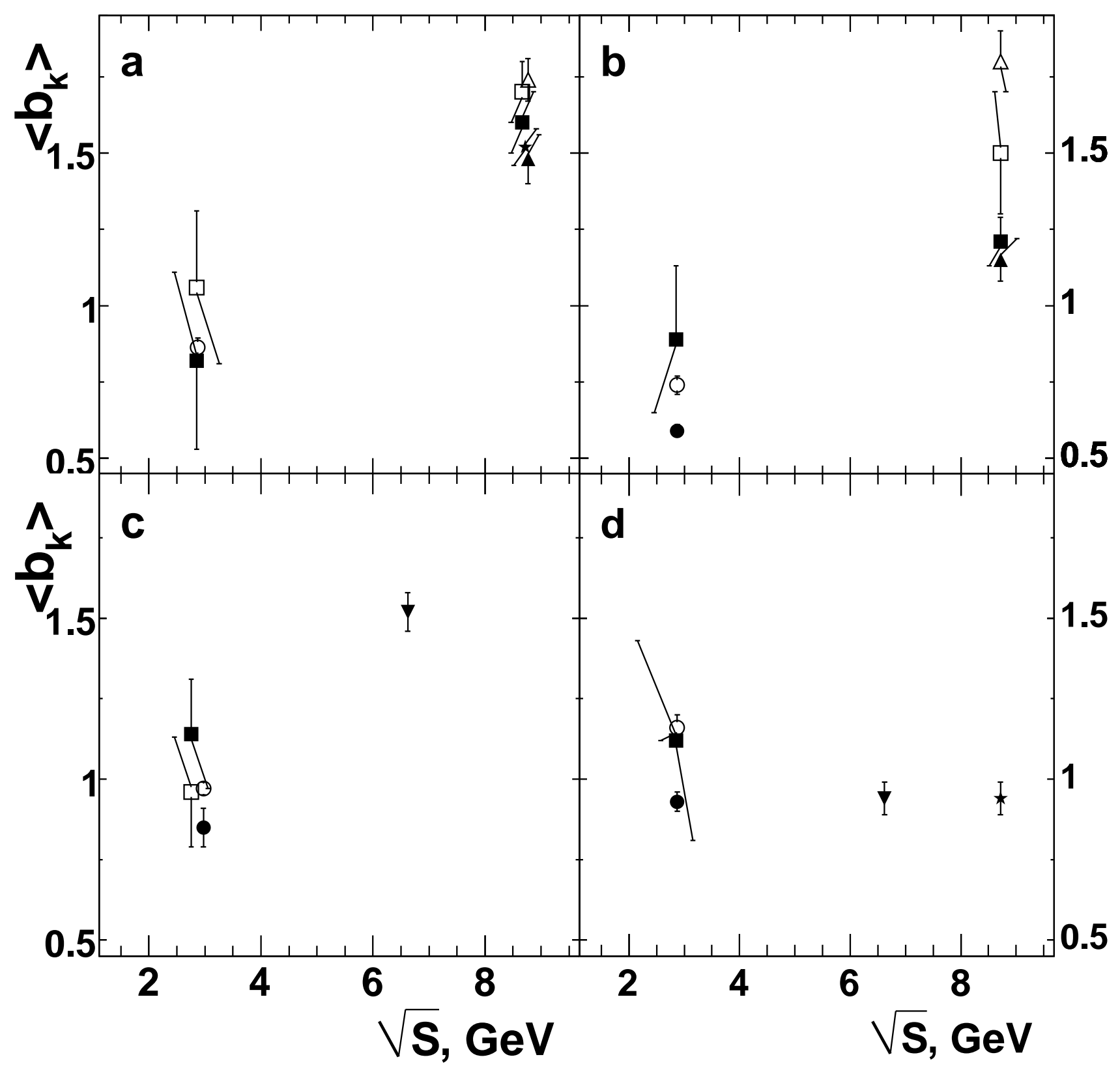

FIG. 2: Values of $\left\langle b_{k}\right\rangle$ versus collision energy for strange particles at $X_{1}=0.1$ (a,b) and $X_{1}=0.2$ (c,d). Left column corresponds to $K^{0}$-mesons (a,c), right column - to $\Lambda^{0}$-hyperons (b,d). Experimental points for target (beam) fragmentation region are marked as follows: - (०) - reaction (2), $\boldsymbol{\nabla}-\overline{\mathrm{p} p}$ at $22.4 \mathrm{GeV} / \mathrm{c}$ [7, [8] $\star-\operatorname{pp}$ at $40 \mathrm{GeV} / \mathrm{c}[8], \boldsymbol{\Delta}(\triangle)-\pi^{-} \mathrm{p}$ at 40 $\mathrm{GeV} / \mathrm{c}[8], \mathbf{\square}(\square)-\pi^{-}\left(\mathrm{C}_{2} \mathrm{~F}_{5} \mathrm{Cl}_{3}\right), \pi^{-} \mathrm{C}$ at 3.9 and $40 \mathrm{GeV} / \mathrm{c}$ [8], respectively. 


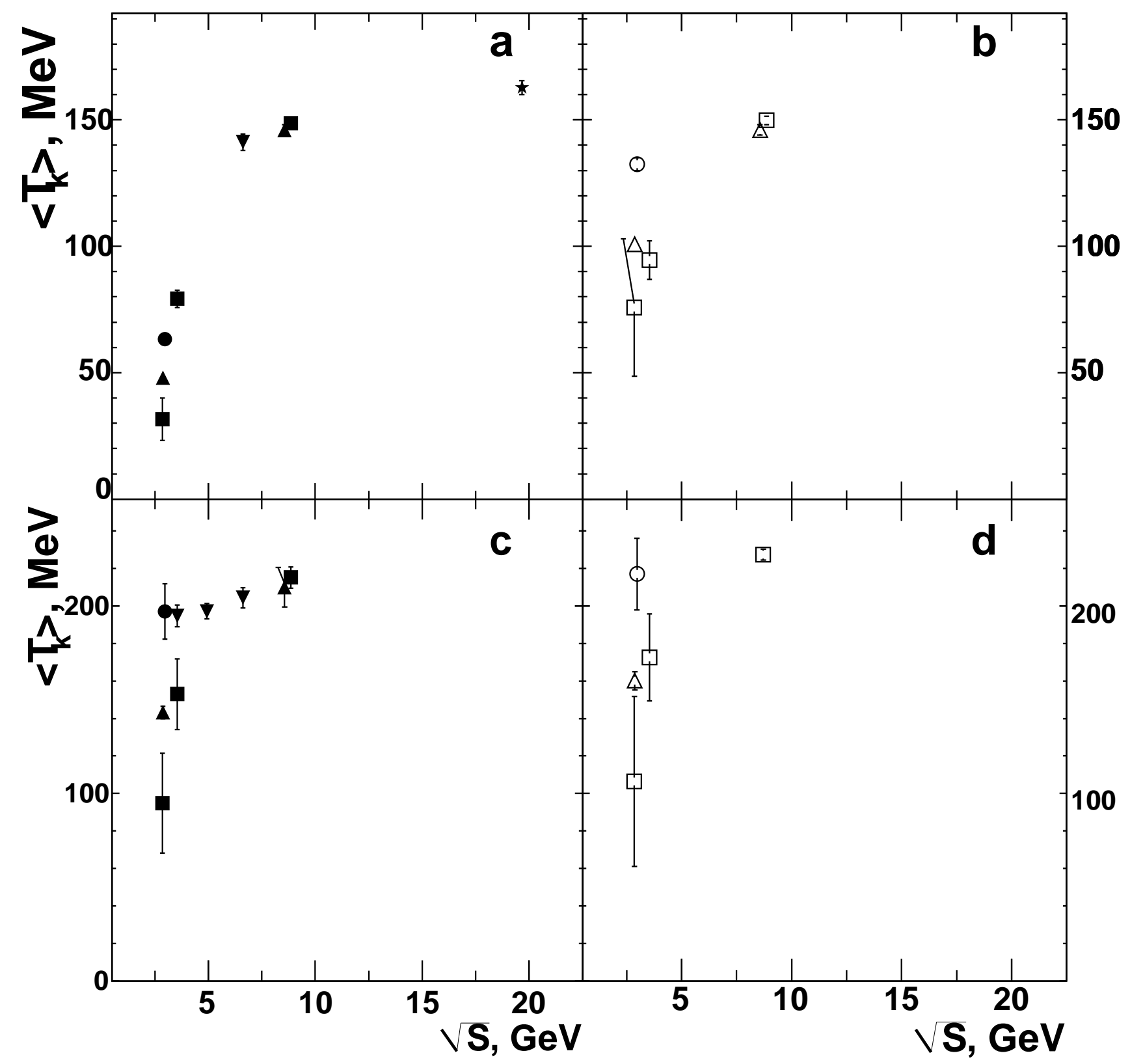

FIG. 3: Dependence $\left\langle T_{k}\right\rangle(\sqrt{s})$ for $\pi^{-}$-mesons at $X_{1}=0.1(\mathrm{a}, \mathrm{b})$ and $X_{\mathrm{l}}=0.2(\mathrm{c}, \mathrm{d})$. Left column corresponds to the target fragmentation region $(\mathrm{a}, \mathrm{c})$, right column - to the beam fragmentation (b,d). Experimental points for target (beam) fragmentation region are marked as follows: $\bullet(\circ)-\pi^{+} \mathrm{p}$ at $4.2 \mathrm{GeV} / \mathrm{c}, \boldsymbol{\nabla}-\overline{\mathrm{p}} \mathrm{p}$ at $5.7,12$ and $22.4 \mathrm{GeV} / \mathrm{c}, \star-\mathrm{pp}$ at $205 \mathrm{GeV} / \mathrm{c}, \boldsymbol{\Delta}(\Delta)-\pi^{-} \mathrm{p}$ at 3.9 and $40 \mathrm{GeV} / \mathrm{c}, \boldsymbol{\square}(\square)-\pi^{-}\left(\mathrm{C}_{2} \mathrm{~F}_{5} \mathrm{Cl}_{3}\right), \pi^{-} \mathrm{Ne}, \pi^{-} \mathrm{C}$ at $3.9,6.2$ and $40 \mathrm{GeV} / \mathrm{c}$, respectively. 


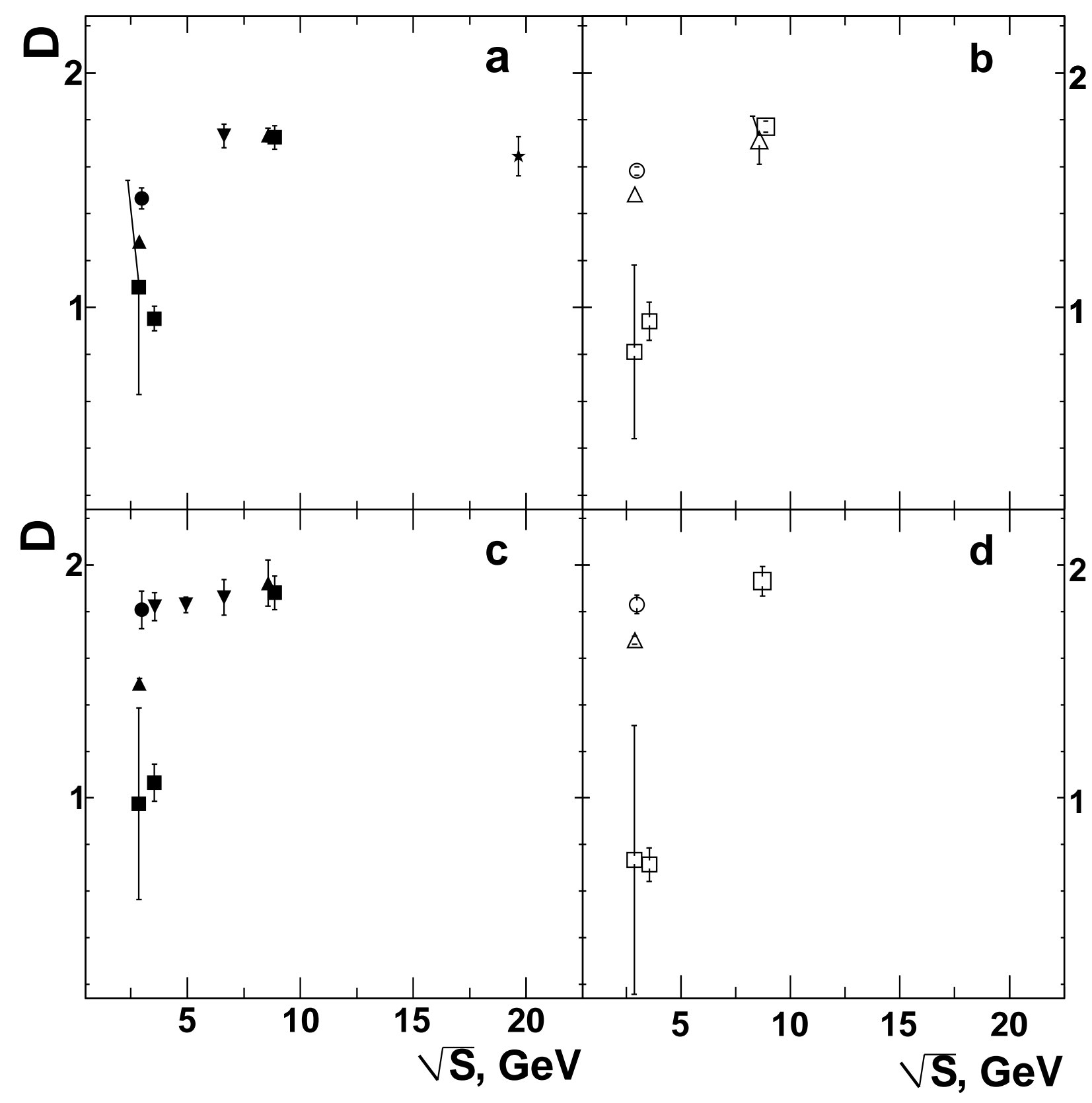

FIG. 4: Dependence of the cluster dimension $D$, calculated for $\pi^{-}$-mesons, on collision energy at $X_{1}=0.1$ (a,b) and $X_{1}=$ $0.2(\mathrm{c}, \mathrm{d})$. Left hand corresponds to the target fragmentation region $(\mathrm{a}, \mathrm{b})$, right hand - to the beam fragmentation (b,d). Experimental points for target (beam) fragmentation region are marked as follows: $\bullet(\circ)-\pi^{+} \mathrm{p}$ at $4.2 \mathrm{GeV} / \mathrm{c}, \boldsymbol{\nabla}-\overline{\mathrm{p} p}$ at 5.7 , 12 and $22.4 \mathrm{GeV} / \mathrm{c}, \star-$ pp at $205 \mathrm{GeV} / \mathrm{c}, \boldsymbol{\Delta}(\triangle)-\pi^{-} \mathrm{p}$ at 3.9 and $40 \mathrm{GeV} / \mathrm{c}, \mathbf{\square}(\square)-\pi^{-}\left(\mathrm{C}_{2} \mathrm{~F}_{5} \mathrm{Cl}_{3}\right), \pi^{-} \mathrm{Ne}^{-} \pi^{-} \mathrm{C}$ at $3.9,6.2$ and $40 \mathrm{GeV} / \mathrm{c}$, respectively. 